\title{
ARQUEOLOGÍA FORENSE Y SUS DIÁLOGOS CON LA ANTROPOLOGÍA SOCIAL EN EL MÉXICO DE LA GUERRA CONTRA LAS DROGAS
}

\author{
FORENSIC ARCHAEOLOGY AND ITS DIALOGUES WITH SOCIAL \\ ANTHROPOLOGY IN THE MEXICO OF THE WAR ON DRUGS
}

\section{ARQUEOLOGIA FORENSE E SEUS DIÁLOGOS COM A AMTROPOLOGIA SOCIAL NO MÉXICO DA GUERRA ÀS DROGAS}

Alejandro Arteaga Saucedo Licenciado en Arqueología Universidade Nacional Autônoma do México errantearteaga@gmail.com

\section{Resumen}

En el México de la guerra contra las drogas, la arqueología se ha unido a la tarea de colaborar con el sistema de impartición de justicia y los colectivos de familiares de personas desaparecidas, pero sus aportes han sido, en su mayoría, más metodológicos que explicativos. Si retomamos el principio de que la arqueología convencional estudia el comportamiento de las sociedades humanas del pasado, y lo aplicamos en el campo forense, podríamos ampliar las posibilidades de investigación o intervención de los contextos de interés forense.

En el artículo se retoman los aportes de la arqueología en la localización de personas desaparecidas y la participación de los colectivos de familiares realizando las mismas tareas y cómo ambos pueden contribuir en la investigación criminal. La finalidad es discutir y proponer la aplicación de los fundamentos teórico-interpretativos de la antropología para la implementación de una arqueología colaborativa que contribuya a la búsqueda de verdad y justicia en contextos contemporáneos de conflicto. Finalmente se retoman los aportes del Grupo de Investigaciones en Antropología Social y Forense para la co-construcción de conocimiento y políticas públicas que cumplan con las expectativas de los colectivos de familiares de personas desaparecidas.

Palabras clave: arqueología, forense, colaborativa, violencia. 


\begin{abstract}
In the Mexico of the war on drugs, archeology has joined the task of collaborating with the justice system and the collectives of relatives of disappeared persons, but their contributions have been, for the most part, more methodological than explanatory. If we take the principle that conventional archeology studies the behavior of human societies of the past, and applied in the forensic field, we could expand the scope of investigation or intervention contexts of forensic interest.

In this article we return to the contributions of archeology in the location of missing persons and the participation of collectives of relatives of missing persons performing the same tasks and how both can contribute to the criminal investigation. The purpose is to discuss and propose the application of the theoretical-interpretative foundations of anthropology for the implementation of a collaborative archeology that contributes to the search for truth and justice in contemporary contexts of conflict. Finally, we will return to the contributions of the Social and Forensic Anthropology Research Group for the co-construction of knowledge and public policies that reach the expectations of the collectives of relatives of disappeared persons.
\end{abstract}

Key words: archaeology, forensic, collaborative, violence.

\title{
Sumario
}

No México da guerra contra as drogas, a arqueologia juntou-se à tarefa de colaborar com o sistema judiciário e os coletivos de parentes de pessoas desaparecidas, mas suas contribuições foram, em sua maior parte, mais metodológicas do que explicativas. Se voltarmos ao princípio de que a arqueologia convencional estuda o comportamento das sociedades humanas do passado e as aplica no campo forense, poderíamos ampliar as possibilidades de investigação ou intervenção de contextos de interesse forense.

No artigo, as contribuições da arqueologia na localização de pessoas desaparecidas e a participação dos grupos de parentes que desempenham as mesmas tarefas e como ambos podem contribuir na investigação criminal são retomadas. O objetivo é discutir e propor a aplicação dos fundamentos teórico-interpretativos da antropologia para a implementação de uma arqueologia colaborativa que contribua para a busca da verdade e da justiça em contextos contemporâneos de conflito. Por fim, são retomadas as contribuições do Grupo de Pesquisa em Antropologia Social e Forense para a co-construção de conhecimentos e políticas públicas que atendam às expectativas dos grupos de familiares de pessoas desaparecidas.

Palavras-chave: arqueologia, forense, colaborativa, violência. 
a arqueología forense en

México surgió en la década de 1990 (Lagunas y Reyes, 2009;

Lara, 2009; Talavera et al., 1999; Talavera y Rojas, 2006; Valencia y Methadzovic, 2009) como un recurso de la antropología física forense que contribuye a la búsqueda, registro y recuperación de restos humanos inhumados clandestinamente o que fueron localizados sobre la superficie del terreno y cuyo estado de conservación complica la identificación positiva de el o los individuos (Dupras et al., 2011; Haglund, 2001; Hunter y Cox, 2005; Lagunas, 2006; Lagunas y Reyes, 2009; Lara, 2009).

Desde entonces, las herramientas de prospección, excavación y registro han contribuido, desde un enfoque técnicodescriptivo, a la investigación criminal o de las violaciones a los derechos humanos (Dupras et al., 2006; Sanabria, 2008). Sus aportes se han adaptado a las propias necesidades del sistema de impartición de justicia, el cual requiere una opinión técnica, basada en una investigación científica, con una o varias hipótesis que son contrastadas con la evidencia material o indicios recuperados en campo.

\section{Interpretando el pasado}

Pero ¿Eso es todo lo que la arqueología puede aportar a la investigación forense?
Veamos. La arqueología "convencional", o la que se ocupa del pasado no tan reciente, retoma los modelos teórico-explicativos de la teoría antropológica para interpretar sus hallazgos. La definición clásica y conocida popularmente afirma que es "el estudio del hombre a partir de sus restos materiales" (Renfrew y Bahn, 2007). Y ¿Qué se estudia del hombre? Su organización social, política y económica, sus modos de subsistencia, el desarrollo tecnológico, las expresiones simbólico-religiosas, la evolución de la sociedad, las dinámicas de población, los conflictos, las condiciones de salud, entre otros ¿Y cuáles son sus restos materiales? Generalmente se componen de fragmentos de cerámica, huesos, rocas trabajadas, manifestaciones artísticas, vidrio, metal, arquitectura, espacios de trabajo, restos orgánicos y la interacción con el medio ambiente.

La arqueología tiende a inferir las acciones del hombre a partir de lo que los restos materiales le pueden proporcionar. Sin embargo, el estudio del pasado va más allá de la descripción de objetos, y se aproxima al comportamiento humano desde los gestos (Duday, 1997), o actos demostrados como intencionales que se reflejan en el contexto en el cual se encuentran. Se entiende como contexto a la relación espacial y temporal que mantienen un grupo de objetos $\mathrm{y}$ materiales orgánicos que resultan de la 
actividad del hombre. El hallazgo de un fogón, carbón, huesos de animal, restos de comida y enseres empleados para la elaboración de alimentos, por ejemplo, conforman un contexto típico de una cocina.

Una muestra de lo anterior es el fascinante caso de la identificación de costumbres funerarias entre los Neandertales y Sapiens de la edad de piedra, en donde el hallazgo de polen de flores en una tumba donde se depositaron uno o varios individuos permitió reconocer el gesto funerario (Duday, 1997) de dejar una ofrenda a los difuntos hace 12,000 años en Eurasia (Rendu et al., 2014). Con esto fue posible suponer la existencia de un complejo sistema de creencias de un grupo que se creía "primitivo".

A pesar de contar con datos plenamente registrados y recuperados en su contexto original, las inferencias hechas por el investigador no dejan de ser, precisamente, interpretaciones, es decir, asunciones hechas a partir de la correlación entre un conjunto de datos. Este asunto ya fue abordado en la teoría arqueológica por décadas, primero, con la aplicación del método científico propuesto por la "Nueva Arqueología" o "Arqueología procesual" (Binford, 2004). Seguido de la crítica a los procesos de construcción y reconstrucción del contexto arqueológico y sus repercusiones en la interpretación de los datos (Schiffer, 1972); y más recientemente, con la a apertura a corrientes "postprocesuales" o "postmodernas" (Hodder, 1999; Johnson, 2000; Renfrew y Bahn, 2007), en donde se consiente que los aportes de la arqueología a la reconstrucción de la historia del hombre no pueden limitarse a la creación de catálogos de objetos y bases de datos.

Actualmente, el arqueólogo trabaja con la información que está a su disposición y argumenta las interpretaciones de sus hallazgos a partir de los datos provenientes del contexto arqueológico. Limitarnos a afirmar que un hueso humano presenta marcas de corte provocadas intencionalmente con un objeto, negaría la oportunidad de analizar los conflictos interpersonales y su influencia en la vida política, religiosa y cotidiana en un momento histórico dado. Para ello será necesario ampliar el panorama e insertar cuidadosamente los hallazgos en su propio contexto espacial, temporal, político $\mathrm{y}$ social. Para hacerlo resultará necesario estudiar más que el hueso o conjunto de huesos.

\section{Interpretando el pasado reciente}

Ahora bien, se define tradicionalmente a la arqueología forense como: "la aplicación de 
la teoría y métodos de la arqueología para la excavación y registro de la escena del crimen"1 (Dupras et al., 2006: 3).

Como se ha dicho anteriormente, este campo del conocimiento usualmente se limita a adaptar las técnicas tradicionales de búsqueda, excavación, liberación, registro y recuperación de los indicios relacionados con un posible hecho criminal. Siguiendo estos pasos se construye una explicación lógico-racional, cuya hipótesis a corroborar es:

- Que el hallazgo de restos humanos no identificados, de los que se desconozca su procedencia o causas, forma parte de un hecho criminal (Sanabria, 2008: 72).

Para comprobar tal hipótesis es necesario responder las siguientes preguntas:

- ¿Dónde se encuentran los restos?

- ¿Son humanos o de animal?

- ¿Son antiguos o recientes?

- ¿De cuántos individuos son?

- ¿Fue un hecho intencional o accidental?
Si se comprueba que se trató de un hecho criminal, las siguientes preguntas a responder son:

- ¿Cómo sucedió el hecho o hechos?

- ¿Cuándo fue?

- ¿Qué del contexto nos ayuda a identificar a la(s) víctima(s)?

- ¿Qué del contexto nos ayuda a identificar al(los) perpetrador (es)?

Resolver estos cuestionamientos resultará suficiente para aportar a la indagación criminal desde la pericia del arqueólogo o arqueóloga. Los indicios finalmente formarán parte de una carpeta de investigación que incluirá los aportes de todos los especialistas involucrados en el caso.

Sin embargo, y de acuerdo con E. Blair (2005: 35):

\begin{abstract}
"los alcances de la antropología van más allá de lo técnico, su reflexión no se reduce a mencionar el hecho de devolverle al muerto su nombre, su historia y su pertenencia a un grupo y por tanto su dignidad, sino que trabaja el problema que viven los más afectados por este hecho: las familias, a quienes se les niega el derecho de recibir un cuerpo para darle sepultura;
\end{abstract}

\footnotetext{
${ }^{1}$ Traducción del autor de: "the application of archaeological theory and methods to crime scene
} 
la población civil en general, y los mismos combatientes".

Es decir, el aspecto social del crimen debe ser analizado bajo el estudio multidisciplinario de las ciencias antropológicas, brindando un análisis no solamente lógico-racional, sino holístico, debido a que se pueden analizar todas las partes que lo componen y conforman como actividad ilegal. De manera tal que la antropología social, en conjunto con la arqueología y la antropología física, deberían lograr la comprensión e interpretación del hecho delictivo, partiendo de aspectos técnicos $\mathrm{y}$ metodológicos para más adelante entender cuestiones de carácter histórico y social.

Se propone, a manera de hipótesis, que mediante el análisis del contexto forense es posible diferenciar, fenómenos naturales y/o accidentales, de comportamientos intencionales entorno a la muerte. Estos últimos son reconocibles a través de la manera en que se trata y colocan los restos de personas asesinadas.

Para reconocer esta clase de comportamientos, el contexto como tal, y no solo los restos humanos, será el elemento principal de una investigación arqueológica forense durante las labores de campo.
La interpretación del contexto, en estos casos, será posible mediante el análisis de la relación espacial que mantiene el cadáver con los objetos asociados a él, los rasgos naturales y culturales que lo rodean, y la manera en que el cuerpo fue manipulado y depositado.

El argumento de dicha suposición sostiene que la muerte del ser humano es un hecho trascendental dentro de las relaciones sociales humanas y representa una ruptura en el orden de la vida. Prácticamente todas las culturas del mundo, desde hace miles de años, someten a sus muertos, amigos o enemigos, a un ritual que les permite sobrellevar ese rompimiento del orden. Ya sea para despedirse, para apropiarse de la identidad del otro o para eliminar simbólicamente al enemigo (Blasco, 2009; Morin, 2007; Parker, 2008; Viveiros De Castro, 2010).

Tal como lo expone E. Blair (2005), en América Latina, la necesidad de incorporar antropólogos físicos y arqueólogos además estuvo acompañada por antropólogos sociales. Estos últimos más allá de estudiar los restos materiales, se interesaron por las graves violaciones de Derechos Humanos que resultaron de las prácticas sistemáticas de desaparición de opositores, comunidades marginales y guerrilleros, que fueron ejercidas por dictaduras militares $\mathrm{y}$ 
gobiernos opresores en países como Argentina, Perú, Colombia o Guatemala desde la década de 1980 (Congram Y Fernández, 2006; Parra Y Palma, 2005; Rodríguez, 1994; Salado Y Fondebrider, 2008; Sanabria, 2008).

Hasta aquí, y tomando en cuenta el contexto sociopolítico dentro del cual surge la antropología forense en Latinoamérica, resulta posible reconocer dos enfoques para estudiar el pasado reciente, cada uno con las siguientes peculiaridades:

1) La búsqueda judicial, la cual se desarrolla en el ámbito técnico-legal y busca sanciones para los presuntos responsables, por lo que frecuentemente se desarrolla en espacios institucionales del sistema de impartición de justicia. Su marco teórico-metodológico tiende a ser hipotético-deductivo y a emplear variables cuantitativas (Ortega, 2017). Debido a los objetivos que pretende cumplir, suele limitarse a la generación de informes y peritajes técnicos que se integran a un proceso judicial (Olarte-Sierra y Castro, 2019).

\section{2) La búsqueda humanitaria, cuya finalidad} es ayudar a las víctimas desde la generación de conocimiento científico comprometido socialmente. Por su naturaleza, frecuentemente es ejercida desde el ámbito académico-colaborativo (Hernández, 2017;
Olarte-Sierra Y Castro, 2019; Querales, 2018). Comúnmente usa un marco teórico hipotético-inductivo y de carácter cualitativo, aunque también incluye metodologías cuantitativas (Ortega, 2017). Su objetivo es acompañar a los familiares en búsqueda y auxiliar en la reconstrucción del pasado y en las exigencias de justicia y verdad, mediante el activismo político y el análisis del contexto sociohistórico del conflicto y de los procesos de marginación y exclusión. Los productos que resultan de este tipo de investigación, pueden ser peritajes socio-antropológicos, producciones audiovisuales y otros recursos científico-académicos (Hernández, 2017; Querales, 2018; Robledo et al., 2016, Robledo et al., 2018).

En los siguientes apartados, se retoman los aportes de la búsqueda humanitaria en el marco de la Guerra contra las drogas (20062019).

\section{Víctimas de la Guerra contra las drogas}

Podría considerarse a la "Guerra sucia" como el antecedente inmediato de la "Guerra contra las drogas". La primera se llevó a cabo entre las décadas de 1970 y 1990 y tenía la finalidad de enfrentar, mediante el uso de la fuerza, a personas o colectivos (armados o no) que ponían en peligro el monopolio del poder y los 
intereses del Estado (Robledo, 2015b; Mastrogiovanni, 2014).

La segunda, conocida como "Guerra contra las drogas", comienza en el año 2006 y da continuidad a las prácticas sistemáticas de desaparición y asesinato llevadas a cabo durante las décadas previas. En este nuevo episodio de la violencia en México, el despliegue de las fuerzas militares en las calles se llevó a cabo por instrucciones del expresidente la república, el C. Felipe Calderón (2006-2012). Se implementó como una "política de seguridad nacional que incluye la intervención militar y policiaca del gobierno federal, así como las acciones de delincuencia organizada simultaneas y anteriores a dicha intervención" (Robledo, 2015b: 95).

Las cruzadas en contra de la llamada "delincuencia organizada", desestabilizaron el orden que aparente mantenían los grandes cárteles de las drogas, fragmentándolos en pequeñas células que desde entonces compiten por el control de los territorios y los mercados ilegales. Desde entonces, los grupos criminales han desarrollado e implementado mecanismos cada vez más violentos, entre los que se complejiza el fenómeno de la desaparición y exposición pública del enemigo asesinado (Ovalle, 2010).
Es en este momento en el que surge la idea de "delincuencia organizada" como una categoría empleada para referirse al enemigo, al no ciudadano, a quien no tiene derecho a ser considerado como víctima (Robledo, 2015a, 2015b; Olarte-Sierra y Castro, 2019). Muchas veces con el argumento de que esas personas no eran humanas, a juzgar por los actos que éstos llegaban a cometer de manera intencionada. Este discurso, en parte impulsado por las instituciones estatales, sirvió desde el inicio para justificar el uso excesivo de la fuerza por parte de las autoridades civiles (locales, estatales y federales) y militares.

La realidad, ciertamente era más compleja de lo que este discurso nos hacía creer: muchos de los supuestos criminales eran jóvenes marginados y con pocas oportunidades provenientes de los barrios conflictivos de las ciudades, campesinos e indígenas que huían de sus comunidades, personas que habían sido despojadas $\mathrm{y}$ deportadas de Estados Unidos durante los últimos años. O, en años más recientes, personas en busca de oportunidades de empleo que eran reclutadas forzosamente por los grupos criminales.

Dentro de este mismo fenómeno, el asesinato de civiles inocentes durante enfrentamientos armados o de personas a las cuales no se les podía comprobar 
relación con el crimen organizado, fue considerado como daño colateral (Robledo, 2015a). Al mismo tiempo que persistió (y persiste) la creencia popular de que los ejecutados y desaparecidos "algo habían hecho" o "en algo andaban". Estos estigmas invisibilizaron a las víctimas de la guerra y deslegitimaron la búsqueda de personas desaparecidas durante los primeros años (Robledo, 2015a, 2015b).

No obstante, con el transcurso de los años comenzó a vislumbrarse la complejidad de las ejecuciones y desapariciones en México (Hernández, 2017; Robledo, 2015a, 2015b). La idea de la "delincuencia organizada", desde los primeros años de la guerra refería por igual a presuntos delincuentes, a grupos sociales e identitarios marginados, indígenas y opositores, que, entre la confusión, formaban parte de las cifras y estadísticas que documentaban las bajas de la guerra.

Entre las víctimas comenzaron a figurar, por igual, migrantes, estudiantes de universidades privadas y públicas, pequeños comerciantes y grandes empresarios, ganaderos y agricultores, defensores de derechos humanos y de los recursos naturales, periodistas y familiares de personas desaparecidas. Algo más estaba ocurriendo.
Desde el sexenio de Felipe Calderón, y luego con su sucesor, quien decidió continuar con esa estrategia de seguridad, el C. Enrique Peña (2012-2018), las noticias en la prensa local y nacional expusieron cada vez más la diversidad y complejidad del fenómeno de la violencia en el país y permitieron reconocer el uso desmedido de la fuerza. El cual, en muchas ocasiones, venía acompañado del despojo y el desplazamiento forzado.

Hasta el día de hoy, resulta confuso adjudicar estos abusos a los grupos criminales o a las instituciones, debido a la oscura fusión que ambos mantienen hasta la actualidad. Casos como el de las fosas comunes de Tetelcingo (Estado de Morelos) en donde el Estado inhumó clandestinamente a personas no identificadas bajo circunstancias sospechosas (Robledo et al., 2016), o el accionar de militares durante el llamado “Operativo Conjunto” Chihuahua (Robledo et al., 2018), permiten percibir la responsabilidad de las instituciones y los exhibe como coproductores de las víctimas que en un principio se atribuyeron exclusivamente a los criminales.

A inicios de 20019, con el cambio de administración, el recién electo presidente de la República, el C. Andrés Manuel López (2018-2024), declaró: "No hay guerra. 
Oficialmente ya no hay guerra, nosotros queremos la paz, vamos a conseguir la paz"2. Esta noticia marcó "oficialmente" el fin del conflicto, sin embargo, la crisis de Derechos Humanos continua: en lo que va del año se han reportado 481 personas desaparecidas y localizado 222 fosas clandestinas, en donde se hallaron 337 cuerpos $^{3}$. Cifras que se suman a los más de 40,000 desaparecidos, 2,000 fosas clandestinas exhumadas y los aproximadamente $26 \mathrm{mil}$ cuerpos $\mathrm{sin}$ identificar que dejaron las administraciones pasadas ${ }^{4}$.

El gobierno entrante propuso enfrentar la situación destinando alrededor de 20 millones de dólares a la Comisión Nacional de Búsqueda, e integrando la opinión y conocimientos de expertos y familiares de personas desaparecidas. De alguna manera presupone un cambio de paradigma, en el que se deja de invisibilizar y estigmatizar a las víctimas y se les toma en cuenta para la creación de políticas públicas que den solución al conflicto. A pesar de ello, la

\footnotetext{
2 Tomado de:

https://www.excelsior.com.mx/nacional/se-acabola-guerra-contra-el-crimen-lopez-obrador/1293646, consultado el 16 de junio de 2019.

${ }^{3}$ Tomado de:

https://aristeguinoticias.com/2805/mexico/en-vivoconferencia-de-prensa-sobre-busqueda-depersonas/, consultado el 29 de mayo de 2019.
}

revictimización y el descontento familiares continúa 5 .

\section{Más perspectivas de aproximación}

La violencia desmedida y las políticas empleadas por el Estado trajeron reacciones de los familiares, quienes se organizaron en colectivo para luchar por los derechos de las víctimas, recuperar a sus seres queridos $\mathrm{y}$ oponerse al discurso oficial que los criminalizaba.

Desde el inicio los colectivos han luchado por recuperar la memoria de los desaparecidos, como una estrategia para generar espacios de reconciliación individual y colectiva (Robledo, 2015a) y como medio para buscar el reconocimiento público de la historia reciente de nuestro país (Robledo, 2014).

Desde entonces, decenas de colectivos, principalmente conformados por mujeres, han salido a los espacios públicos para exigir el regreso con vida de sus seres queridos. Estas búsquedas no son nuevas en el país, ya que, desde por lo menos la década de 1960, el Estado enfrenta acusaciones por

\footnotetext{
${ }^{4}$ Tomado de: https://www.gob.mx/segob/prensa/segob-enbusqueda-de-mas-de-30-mil-personasdesaparecidas-en-mexico?idiom=es, consultado el 16 de junio de 2019.

5 Tomado de: https://www.razon.com.mx/mexico/abandonanjalisco-y-sonora-a-comisiones-de-busqueda/, consultado el 17 de junio de 2019.
} 
secuestrar y desaparecer a activistas, minorías y opositores. Incluso desde entonces se tiene la sospecha de la presencia de cementerios clandestinos en varios campos militares del país 6 .

El fenómeno actual es distinto. La violencia generalizada, la presencia activa de grupos de criminales organizados y la supuesta guerra emprendida por el Estado en contra de los criminales, ha dejado una ola de alrededor de 40,000 personas desaparecidas durante los últimos doce años $\mathrm{y}$ una cantidad mucho mayor de familias afectadas ${ }^{7}$.

La poca contribución de las autoridades en las búsquedas y la desesperación de los familiares por obtener respuestas los ha llevado a buscar más allá de los hospitales, cárceles o morgues, motivándolos a continuar con sus indagaciones en fosas clandestinas, o incluso, en cementerios oficiales. Así, en cuestión de años, y sin quererlo, numerosas madres, esposas y hermanas de todo el país se han convertido en expertas en la localización de fosas clandestinas y en la identificación de restos óseos humanos.
Sus conocimientos, en parte teóricos (adquiridos en talleres, seminarios y cursos impartidos por Organizaciones No Gubernamentales), pero principalmente prácticos, han incomodado a las autoridades y a la comunidad científica, debido a que no respetan los protocolos ni siguen los procedimientos de manera sistemática $\mathrm{y}$ ordenada. Es aquí donde resurgen las preguntas ya planteadas por los mismos familiares: ¿Entonces quién lo hará? ¿Quién más está a dispuesto a arriesgar su vida para recuperar la identidad de los desaparecidos?

La situación, por supuesto, ha sido aprovechada por el mismo Estado, culpando a los familiares por la pérdida de evidencia científica durante la exhumación de fosas clandestinas.

La experiencia acumulada por los colectivos de familiares, como en el caso de la arqueología forense, va más allá de los métodos y técnicas para la localización de fosas clandestinas. Con el paso de los años han podido reflexionar y documentar las dimensiones sociales, históricas, políticas y de poder que están detrás de la desaparición de personas. Han tenido la oportunidad de analizar las causas y consecuencias de la

\footnotetext{
7 Tomado de: https://www.eleconomista.com.mx/politica/EnMexico-suman-mas-de-40000-desaparecidos20190118-0003.html, consultado el 26 de febrero de 2019.
}

\footnotetext{
${ }^{6}$ Tomado de:

https://www.eluniversal.com.mx/articulo/estados/2 015/08/16/desaprecidos-guerra-sucia-deja-480victimas6, consultado el 26 de febrero de 2019.
} 
violencia e identificar a los actores y factores históricos, sociales, políticos y económicos que están detrás de la violencia generada durante los últimos años.

Estos conocimientos surgen de preguntarse ¿Qué pasó con sus seres queridos? y una vez emprendida la búsqueda a cuestionarse sobre ¿qué buscar? ¿cómo hacerlo? y ¿qué tipo de hallazgos deberían ser documentados? Las respuestas los han motivado a crear sus propias metodologías y a desarrollar estrategias de búsqueda y recuperación propias.

Más allá de eso, el familiar se convirtió en un activista político que sale a tomar los espacios públicos y cuestionar el lenguaje legal, un lenguaje oficial que no necesariamente representa sus intereses y necesidades (Hernández, 2017; Querales, 2018; Robledo, 2014, 2015a, 2015b).

Este discurso oficial promovido por las autoridades se basa en la idea de la ciencia y el método científico, como el único capaz de acceder a la verdad y la justicia. Por lo que, aparte de monopolizar el uso de la fuerza, se controla la producción del conocimiento de la realidad y las maneras de aproximarse al pasado.

La ciencia forense, desde esta perspectiva, busca explicar al mundo objetivamente, "tal y como es" (Olarte-Sierra y Castro, 2019).
Pero "sin reconocer que sus intervenciones ayudan a interpretar y dar forma a ese mismo pasado que intentan presentar como neutral" (Olarte-Sierra y Castro, 2019). A la vez que desestima las múltiples experiencias de las víctimas, y oscurece otros procesos y verdades; haciendo que los familiares descalifiquen sus propios saberes y maneras de acceder a la realidad (Ferrandiz, 2010). A pesar de que son ellos quienes han experimentado la crueldad y el horror de la violencia (Querales, 2018).

En ese sentido, son los colectivos de familiares de personas desaparecidas quienes, desde su perspectiva, reconocen otros gestos en el actuar del criminal y están más familiarizados con el contexto social, histórico y político detrás de la desaparición de personas; $y$ han reflexionado entorno a los múltiples factores que rodean a la desaparición y las consecuencias que ésta trae a su comunidad.

\section{Hacer arqueología desde otras perspectivas}

En la teoría antropológica de los últimos años, esta problemática ya ha sido abordada. Se ha planteado la posibilidad de descolonizar el pensamiento teórico, escuchar las voces excluidas o marginadas y co-construir conocimiento científico con los actores sociales con los que la antropología interactúa (Hernández, et al., 
2013; Jimeno, 2000; Sousa, 2010, 2012; Suárez y Hernández, 2008; Viveiros De Castro, 2013).

Estos enfoques parten de criticar las nociones convencionales $\mathrm{u}$ oficiales de ciencia, derecho, persona y justicia. Las cuales son entendidas como categorías que no consideran la pluralidad del ser y estar en el mundo (Hernández, 2011, Hernández, et al., 2013; Jimeno, 2000; Sousa, 2010, 2012; Viveiros De Castro, 2013).

En cuanto a la búsqueda humanitaria en el México de la Guerra contra las drogas, las perspectivas descolonizadoras en antropología y etnografía ya han hecho su aporte, con el objeto de visualizar a las víctimas y replicar sus voces en espacios públicos y foros académicos (Hernández, 2017; Querales, 2018; Robledo, 2014, 2015a, 2015b). Mientras que la arqueología forense mexicana se ha limitado a la búsqueda judicial, haciendo de lado a las experiencias de búsqueda generadas por los colectivos de familiares de personas desaparecidas (Lara, 2009; Talavera et al., 1999; Talavera y Rojas, 2006).

Actualmente, en el gremio a nivel internacional, los estudios sobre la violencia y el conflicto se han enfocado en dos intereses principales: el primero, la violencia interpersonal en el pasado (Chávez, 2017, por mencionar un ejemplo actual en la arqueología mexicana) y en la historia reciente (Moshenska, 2009; Passmore et al., 2015). El segundo, más de carácter reflexivo, se centra en cómo los conflictos contemporáneos influyen en la investigación arqueológica sobre el pasado, principalmente en países de Medio Oriente como Irak, Irán o Siria. Las reflexiones giran en torno a los daños al patrimonio histórico y lo complicado que resulta realizar trabajo de campo en estos lugares (Pollock, 2016). Poco o nulo interés tienen los contextos de violencia actuales o de la historia reciente, a pesar de que la arqueología cuenta con herramientas teóricas y metodológicas para hacerlo.

Cabe señalar que una diferencia fundamental entre la arqueología que estudia la violencia en el pasado y la arqueología forense, es que en la segunda debemos dejar de conceptualizar al cadáver como objeto y comprenderlo como persona (Hanson, 2007: 8). Es decir, en las ciencias forenses cambia la manera en que los mismos restos materiales son percibidos, ya que la fascinación e interés que genera en el investigador el hallazgo de un tzompantli, o pila de cráneos de sacrificados al inicio del periodo colonial en Mesoamérica (Díaz Del Castillo, 2005 [1632]), se convierte en horror cuando se trata del hallazgo de una fosa clandestina contemporánea. 
Al respecto, se podría considerar que las diferencias entre un tipo de arqueología y otro, son: la ética del tratamiento del cuerpo, el tener que trabajar en comunidades bajo estrés, las implicaciones legales y de los Derechos Humanos y lo perturbador de la evidencia recuperada (Hanson, 2007). Sin embargo, las distinciones se amplían cuando esta labor se ejerce en "en casa” (Julián, 2010), es decir, cuando se indaga en el campo de la vida cotidiana del propio investigador (Querales, 2018). Particularmente en el caso mexicano, donde el conflicto se mantiene vigente, destacan las siguientes peculiaridades: 1) Colaborar activamente con los colectivos de familiares de personas desaparecidas; 2) Indagar sobre el pasado reciente de la comunidad de la cual se es miembro; 3) Hacer trabajo de campo en territorios que se mantienen en disputa por los distintos actores armados; 4) La desconfianza en las autoridades; 5) Convivir con los creadores de las fosas, como lo haría cualquier otro actor social que forma parte de esta realidad.

Desde experiencias similares, destaca el trabajo de Y. Hamilakis, quien aborda los conflictos actuales en el Medio Oriente (Hamilakis, 2003, 2009) y los refugiados que huyen de la guerra (Hamilakis, 2015,

\footnotetext{
${ }^{8}$ Traducción propia de: "understanding their own material and temporal sensitivities, their own
}

2018). En sus obras plantea una reflexión teórica y ética respecto a la descolonización del pensamiento arqueológico y la posibilidad de dialogar con los agentes creadores de los contextos arqueológicos. En sus propias palabras, dar voz a los actores sociales ayuda a "comprender sus propias sensibilidades materiales $\mathrm{y}$ temporales, sus propias percepciones $\mathrm{y}$ prácticas en tiempo y forma, sus propias arqueologías"8 (Hamilakis, 2016: 4).

A la aplicación de estos postulados teóricos y políticos en el estudio del pasado se le conoce como "arqueología colaborativa", la cual centra su interés en las taxonomías y ontologías locales, involucrando a las comunidades en el proceso de generar conocimiento (Cipolla et al., 2019). Sus principales aplicaciones se centran en la creación de clasificaciones locales de suelos, plantas y otros elementos del entorno natural y el construido socialmente, además de la co-creación de políticas públicas para la protección y difusión del patrimonio (Cipolla et al., 2019; Nicholas et al., 2011; Owoc, 2006; Rhoads, 1992).

La colaboración con las comunidades locales permite poner en duda las interpretaciones sobre el pasado. Entre las que destacan: 1) La relación entre lo own archaeologies" (HAMILAKIS, 2016: 4). 
material y lo inmaterial. Por ejemplo, los rasgos naturales no modificados, como cerros o formaciones rocosas, ahora son considerados dentro del inventario de yacimientos arqueológicos (AlmagroGorbea y Gari, 2017); 2) La codependencia entre el humano y su entorno. Ahora se pone en duda la posición del hombre como explotador del medio geográfico y se revela la agencia de las cosas y los animales y la compleja relación que mantienen unos con otros (Ingold, 2000); 3) La dicotomía pasado-presente. Las comunidades indígenas ponen en duda la idea de que los artefactos pertenecen al pasado, afirmando que conservan su agencia y forman parte de la realidad actual (Cipolla et al., 2019).

\section{Hacia una arqueología colaborativa en contextos de conflicto contemporáneo}

En los apartados anteriores se resumen los avances en la investigación colaborativa entre la arqueología y las comunidades con las cuales interactúa, sobre todo para el estudio del pasado. Al mismo tiempo se expuso el poco trabajo realizado en cuanto al estudio de los contextos de conflicto actuales. La finalidad de lo anterior es explorar las posibilidades teóricas y prácticas de realizar trabajo colaborativo con colectivos de familiares de personas desaparecidas en el contexto de la violencia generada por la guerra contra el crimen en
México. La experiencia del Grupo de Investigaciones en Antropología Social y Forense (GIASF) ha sido enriquecedora en ese sentido.

Desde sus inicios el GIASF, mantiene el interés por compartir y generar conocimiento científico de la mano de los colectivos de familiares. Es por ello que desde el 2016 imparte talleres en los Estados de Ciudad de México, Morelos, Veracruz, Coahuila, Sinaloa, Tijuana, Chihuahua y Nuevo León y colabora en la creación de peritajes e informes Hernández, 2017; Querales, 2018; Robledo et al., 2016, Robledo et al., 2018; Giasf, 2018).

Los talleres han contribuido a que los colectivos de familiares puedan fortalecer sus experiencias, reconstruir sus saberes, sistematizar su información y adquirir nuevos conocimientos en materia de antropología física, genética, derecho penal, arqueología, etnografía y trabajo de campo (Giasf, 2018).

La experiencia resulta enriquecedora en ambos sentidos, ya que, por otro lado, los conocimientos de los colectivos desestabilizan los conceptos de la ciencia dura y fomentan la creación de conocimientos útiles para analizar las causas y consecuencias de la violencia e identificar a los distintos actores que la generan y reproducen, entre ellos las 
instituciones que se han ocupado de revictimizar a quienes tienen un familiar desaparecido.

En el proceso de compartir conocimientos, también nos hemos convertido en activistas políticos que comparten el reclamo de los familiares para luchar en los espacios donde se hacen las políticas públicas, con la finalidad de buscar que las experiencias de las víctimas sean escuchadas y tomadas en cuenta para implementar una búsqueda que cumpla con sus expectativas de justicia, reparación y no repetición.

Hacer trabajo de campo, arqueológico o etnográfico, en contextos de conflicto vigente, y haciendo antropología en "en casa" (Julián, 2010), facilitan la reflexión no solo como académica o académico, sino como testigo cotidiano y víctima (directa o indirecta). En estos casos no existe la mirada "desde fuera" del fenómeno y la empatía resulta algo fundamental para dejar de ser testigos e intentar formar parte de la solución al problema.

El trabajo llevado a cabo por el GIASF muestra la importancia de la coproducción de conocimiento, para aproximarnos al fenómeno de las desapariciones en el México de la guerra contra las drogas, a partir de las pruebas, testimonios y experiencias que los colectivos de familiares de personas desaparecidas han recuperado durante sus búsquedas (Hernández, 2017; Querales, 2018; Robledo et al., 2016, Robledo et al., 2018, Giasf, 2018).

Ha sido también una gran oportunidad para afrontar el monopolio del conocimiento, la verdad y la memoria (Querales, 2018; Robledo, 2014), de la mano de quienes han vivido la guerra muy de cerca.

Al mismo tiempo, la colaboración entre académicos y colectivos contribuye a buscar otras formas de entender conceptos como contexto, pasado, fosa, cuerpo o cadáver. Una labor necesaria para cumplir con las expectativas que tienen los familiares en búsqueda.

Un ejemplo de ello es el valor que se da a los objetos y la capacidad de comunicarnos con aquello que materializa el horror, pero también la identidad y el amor de una madre por su hijo o hija.

A nivel ético-personal, formar parte del GIASF es una experiencia que muestra que la investigación científica va más allá de las herramientas y métodos, y que el trabajo colaborativo enseña a escuchar, aprender y compartir desde nuestros propios conocimientos y experiencias para reconocer en colectivo la realidad de la cual formamos parte y a la cual no podemos evitar o negar. 
Desafortunadamente, es un campo de estudio complicado y peligroso, basta recordar que los grupos criminales, militares y policías armados continúa desapareciendo y asesinado personas.

La participación de especialistas en arqueología y antropología durante la búsqueda y exhumación de cuerpos en México, está inserta en un contexto de violencia prolongada por años, a diferencia de las experiencias forenses de otros países latinoamericanos como Argentina, Chile, Perú o Guatemala, en donde las tareas de investigación se llevaron a cabo después de que el conflicto había terminado (Congram y Fernández, 2006; Parra y Palma, 2005; Rodríguez, 1994; Salado y Fondebrider, 2008; Sanabria, 2008).

Tal vez debido a eso, pocos arqueólogos en México se involucran en las búsquedas, ya sea contratados por instancias gubernamentales, como voluntarios en los colectivos de familiares o como miembros de grupos independientes de expertos. Pareciera que el gremio arqueológico nacional, prefiere mantenerse al margen, ajenos a lo que se vive día a día.

A pesar de ello, las búsquedas continuarán, al igual que la oportunidad de crear conocimiento en colectivo y de contribuir a cambiar nuestra realidad social desde distintos campos del conocimiento y saberes.

\section{Referências bibliográficas}

ALMARGO-GORBEA, Martín, LACRUZ, Ángel G. (editores). Sacra Saxa: Creencias y ritos en piedras sagradas: Actas del Coloquio Internacional celebrado en Huesca del 25 al 27 de noviembre del 2016. Huesca, Instituto de Estudios Altoaragoneses, 2017.

BINFORD, LEWIS. En busca del pasado. Descifrando el registro arqueológico. Barcelona, Editorial Critica Biblioteca de bolsillo, 2004.

BLAIR, Elsa. Muertes violentas. La teatralización del exceso. Antioquia, Editorial Universidad de Antioquia, 2005.

BLASCO, Diego. La historia de la muerte. Creencias y rituales funerarios. Buenos Aires, LIBSA, 2009.

CHÁVEZ, Ximena. Sacrificio humano y tratamientos postsacrificiales en el templo mayor de Tenochtitlan. Ciudad de México, Secretaría de Cultura-Instituto Nacional de Antropología e Historia, 2017.

CIPOLLA, Craig N., QUINN, James, LEVY, Jay. "Theory in collaborative indigenous archaeology: insights from Mohegan", American Antiquity, v. 84, v. 1, pp. 127-142, 2019.

CONGRAM, Derek, FERNÁNDEZ, Ariana. "Introducción a la antropología y arqueología forense". Cuadernos de Antropología, n. 16, pp. 47-57, 2006.

DIAZ DEL CASTILLO, Bernal. Historia verdadera de la conquista de la Nueva 
España. Ciudad de México, Editorial Porrúa, 2005 [1632].

DUDAY, Henri. “Antropología biológica de "campo", tafonomía y arqueología de la muerte". En: Elsa Malvido, Gregori Pereira y Vera Tiesler (coord.), El cuerpo humano y su tratamiento mortuorio. Ciudad de México: INAH-CEMCA-CONACULTA, 1997, pp. 91-126.

DUPRAS, Tosha L., SCHULTZ, John J., WHEELER, Sandra M., WILLIAMS, Lana J. Forensic Recovery of Human Remains: Archaeological Approaches. Florida, CRC Press, 2006.

DUPRAS, Tosha L., SCHULTZ, John J., WHEELER, Sandra M., WILLIAMS, Lana J. Forensic Recovery of Human Remains: Archaeological Approaches. Segunda edición. Florida, CRC Press, 2011.

FERRANDIZ, Francisco. "De las fosas comunes a los derechos humanos: el descubrimiento de las desapariciones forzadas en la España contemporánea”. Revista de Antropología Social, v. 19, pp. 161-189.

\section{GRUPO DE INVESTIGACIONES EN} ANTROPOLOGÍA SOCIAL Y FORENSE (GIASF). Guía de Búsqueda de personas desaparecidas con enfoque en la verdad y la justicia. Ciudad de México, Colectiva Hermanas en la Sombra, 2018.

HAGLUND, William D. "Archaeology and forensic death investigations".

Historical Archaeology, v. 35, n. 1, pp. 2634, 2001.

HAMILAKIS, Yannis. "Iraq, stewardship, and the 'record': an ethical crisis for archaeology" Public Archaeology, v. 3, n. 2, pp. 104-11, 2003.

HAMILAKIS, Yannis. "The "war on terror' and the military-archaeology complex: Iraq, ethics, and neocolonialism", Archaeological Journal of World Archaeological Congress, v. 5, n. 1, pp. 39-65, 2009.

HAMILAKIS, Yannis. “Arqueología y sensorialidad. Hacia una ontología de afectos y flujos". Vestígios, v. 9, n. 1, pp. 31-53, 2015.

HAMILAKIS, Yannis. "Decolonial archaeologies: from ethnoarchaeology to archaeological ethnography". World Archaeology, v. 48, n. 5, pp. 1-5, 2016.

HAMILAKIS, Yannis. "Decolonial archaeology as social justice". Antiquity, v. 92, n. 362, pp. 518-520, 2018.

HANSON, Ian. "Psycho-social issues and approaches in forensic archaeology". Archaeological Review from Cambridge. v. 22, n. 2, pp. 1-19, 2007.

HERNÁNDEZ, Rosalva, A. "Mexico in times of violence and impunity: legal and forensic anthropology in support of human rights". Portal Web Magazine of Llilas Benson Latin American Studies and Collections. Consultado en: https://lilasbensonmagazine.org/2017/08/2 9/mexico-en-tiempos-de-violencia-eimpunidad-la-antropologia-juridica-y-laantropologia-forense-en-apoyo-a-losderechos-humanos/, 2017.

HERNÁNDEZ, Rosalva A., SIEDER, Rachel, SIERRA, María T. "Introducción". En: SIERRA, María T., HERNÁNDEZ, Rosalva A., SIEDER, Rachel (Eds.). 
Justicias indígenas y Estado. Violencias contemporáneas. Ciudad de México: FLACSO México, 2013, pp. 13-47.

HODDER, Ian. The Archaeological process. An introduction. New Jersey, Blackwell Publishers, 1999.

HUNTER, John, COX, Margaret. Forensic Archaeology: Advances in theory and practice. Londres, Routledge, 2005.

INGOLD, Tim. The perception of the environment. Essays on livelihood, dwelling and skill. Londres, Routledge, 2000.

JIMENO, Miriam. "La emergencia del investigador ciudadano: estilos de antropología y crisis de modelos en la antropología colombiana". En: TOCANCIPÁ, Jairo (Ed.). La formación del Estado nación y las disciplinas sociales en Colombia. Pompayá: Universidad del Cauca, 2010, pp. 157-190.

JOHNSON, Matthew. Teoría arqueológica. Una introducción. Traducción de Josep Ballart. Barcelona, Ariel Historia, 2000.

JULIAN, Gastón. "Etnografía, archivos y expertos. Apuntes para un estudio antropológico del pasado reciente". Revista Colombiana de Antropología, v. 46, n. 2 , pp. 249-278.

LAGUNAS, Zaid. "La antropología física forense, una especialidad necesaria". Ciencia Ergo Sum, v. 13, n. 2, pp. 211-17, 2006.

LAGUNAS, Zaid, REYES, Ana M. "Apuntes para la historia de la antropología física forense en México".
Revista criminalidad, v. 51, n. 2, pp. 6179.

LARA, Israel. Fundamentos de antropología forense: técnicas de prospección, exhumación y análisis de restos óseos en casos forenses. Ciudad de México, INAH, 2009.

MASTROGIOVANNI, Federico. Ni vivos ni muertos, la desaparición forzada en México como estrategia de terror. Ciudad de México: Grijalbo, 2014.

MORIN, Edgar El hombre y la muerte. Quinta edición. Barcelona, Editorial Kairos, 2007.

MOSHENSKA, Gabriel. "Working with memory in the archaeology of modern conflict", Cambridge Archaeological Journal, v. 20, n. 1, pp. 33-48, 2009.

NICHOLAS, George P., ROBERTS, Amy, SCHAEPE, David M., WATKINS, Joe, LEADER-ELLIOT, Lyn, ROWLEY, Susan. "A consideration of Theory, principles and practice in collaborative archaeology", Archaeological Review from Cambridge, v. 26, n. 2, pp. 11-30, 2011.

OLARTE-SIERRA, María F., CASTRO, Jaime E. "Notas forenses: conocimiento que materializa a los cuerpos del enemigo en fosas paramilitares y falsos positivos". Antípoda. Revista de antropología y Arqueología, v. 34, pp. 119-140.

ORTEGA, Albertina. "La antropología forense en los albores del siglo XXI". En: GONZÁLEZ, Lauro, BARRAGÁN, Anabella (Coords.). Antropología física. Disciplina bio-psico-social. Ciudad de México: INAH-ENAH-Mil Libros Editorial, 2017, pp. 61-83. 
OVALLE, Liliana. "Imágenes abyectas e invisibilidad de las víctimas. Narrativas visuales de la violencia en México". Revista El Cotidiano, n. 164, pp. 103-115.

OWOC, Mary A. "Beyond geoarchaeology: pragmatist explorations of alternative viewscapes in the British Bronze Age and beyond". En: ROBERTSON, Elizabeth, SEIBERT, Jeffrey, FERNÁNDEZ, Deepika C., ZENDER, Marc U. (Eds.). Space and spatial analysis in archaeology. Calgary: University of Calgary Press-University of New Mexico Press, 2006, pp. 2-13.

PARKER, Mike. The archaeology of death and burial. Quinta impresión. College Station, A\&M University Press, 2008.

PARRA, Roberto, PALMA, Martha. "Desde el rincón de los muertos y la memoria de sus familiares. Aportaciones forenses de la antropología a los Derechos Humanos". AIBR Revista de Antropología Iberoamericana, n. 39, pp. 1-15, 2005.

PASSMORE, David, HARRISON, Stephan, TUNWELL, David C. "Second World War conflict archaeology in the forest of north-west Europe". Antiquity, v. 88, n. 342, pp. 1275-1290, 2015.

POLLOCK, Susan. "Archaeology and contemporary warfare". Annual Review of Anthropology, v. 45, pp. 215-231, 2016.

QUERALES, May-Ek. “Trabajo colaborativo: un esquicio para el diálogo ético con víctimas de la estrategia de seguridad en México". Acta Sociológica, n. 75, pp. 37-59, 2018.
RENFREW, Colin, BAHN, Paul. Arqueología. Teorías, métodos y prácticas. Tercera edición. Madrid, Akal, 2007.

RENDU, William, BEAUVAL, Cédric, CREVECOEUR, Isabbelle, BAYLE, Priscilla, BALZEAU, Antoine, BISMUTH, Thierry, BOURGUIGNON, Laurence, DELFOUR, Géraldine, FAIVRE, JeanPhilippe, LACRAMPE-CUYAUBRE, François, TAVORMINA, Carlotta, TODISCO, Dominique, TURQ, Alain, MAUREILLE, Bruno. "Evidence supporting an intentional Neandertal burial at La Chapelle-aux-Saints", PNASS, v. 111, n. 1, pp. 81-86, 2014.

RHOADS, James W. "Significant sites and non-site archaeology: a case-study from south-east Australia". World Archaeology, v. 24, n. 2, pp. 198-217, 1992.

ROBLEDO, Carolina. "Espacios sacralizados em las fronteras de la religión: los predios del Pozolero". Vita Brevis, a. 3, n. 5, pp. 79-90, 2014.

ROBLEDO, Carolina. "Un memorial sin memoria: disputas por el reconocimiento de las víctimas en México". Alternativas, no. 5 , pp. 1-31, 2015a.

ROBLEDO, Carolina. "El laberinto de las sombras: desaparecer en el marco de la guerra contra las drogas". Revista de Estudios Políticos, n. 47, pp.89-108, 2015b.

ROBLEDO, Carolina, ESCORCIA. Lilia, QUERALES, May-Ek, GARCÍA, Glendi. "Violencia e ilegalidad en las fosas de Tetelcingo: interpretaciones desde la antropología”. Resiliencia, n. 3, pp. 8-24, 2016. 
ROBLEDO, Carolina, LÓPEZ, Erika L., QUERALES, May-Ek, HERNÁNDEZ,

Rosalva A. Peritaje socio-antropológico sobre el contexto de militarización y graves violaciones de Derechos Humanos cometidas por el ejército en el marco del Operativo Conjunto Chihuahua, especialmente en el norte de Chihuahua y sus impactos comunitarios relacionado con el caso Alvarado Espinoza y otros vs. México, expediente número $\mathrm{CDH}-14$ 2016/011. Inédito, 2018.

RODRÍGUEZ, José. Introducción a la antropología forense. Análisis e identificación de restos óseos humanos. Santafé de Bogotá, Departamento de Antropología de Bogotá, 1994.

SALADO, Mercedes, FONDEBRIDER, Luis. "El desarrollo de la antropología forense en la Argentina". Revista Cuadernos de Medicina Forense, n. 53-54, pp. 213-221, 2008.

SANABRIA, Cesar. Antropología forense y la investigación médico legal de las muertes. Bogotá, Editorial Rasgo y Color, 2008.

SCHIFFER, Michael. "Archaeological context and systemic context", American Antiquity, v. 37, n. 2, pp. 156-165, 1972.

SOUZA, Boaventura de. Derecho y emancipación (Pensamiento Jurídico Contemporáneo n. 2). Quito, Corte Constitucional para el Período de Transición, 2012.

SOUZA, Boaventura de. Descolonizar el saber, reinventar el poder. Montevideo, Ediciones Trilce-Extensión Universidad de la República, 2010.
SUÁREZ, Liliana, HERNÁNDEZ, Rosalva A. (Eds.). Descolonizando el feminismo: teorías y prácticas desde los márgenes. Madrid, Ediciones Cátedra, 2008.

TALAVERA, Jorge A., ROJAS, Martín. "Actualidades de la arqueología y la antropología física forense: un acercamiento intradisciplinario en México para el combate contra el crimen". Diario de Campo, v. 83, pp. 64-87, 2006.

TALAVERA, Jorge A., ROJAS, Martín, CRESPO, Edwin, SÁNCHEZ, Roberto.

"Los peritajes de arqueología y antropología forenses en México: Un nuevo campo de trabajo en las ciencias sociales". Diario de Campo, v. 16, Supl. 4, pp. 12-15, 1999.

VALENCIA, Lorena, METHADZOVIC, A. "La antropología forense en México". Revista Española de Antropología Física, v. 30, pp. 1-9, 2009.

VIVEIROS DE CASTRO. Metafísicas caníbales. Líneas de antropología postestructural, España, Katz Editores, 2010.

VIVEIROS DE CASTRO. La mirada del jaguar. Introducción al perspectivismo amerindio, Argentina, Tinta Limón, 2013. 